\title{
SARS-CoV-2 Neurotropism and Single Cell Responses in Brain Organoids Containing Innately Developing Microglia
}

\section{Samudyata Samudyata}

Karolinska Institutet

Ana Oliveira

Karolinska Institutet https://orcid.org/0000-0003-4119-1096

\section{Susmita Malwade}

Karolinska Institutet

\section{Nuno Rufino de Sousa}

Karolinska Institutet https://orcid.org/0000-0002-0670-9788

\section{Sravan Goparaju}

Karolinska Institutet

\section{Funda Orhan}

Karolinska Institutet

\section{Laura Steponaviciute}

Karolinska Institutet https://orcid.org/0000-0001-8819-0643

\section{Steven Sheridan}

\section{Roy Perlis}

Mass General Brigham

Antonio Rothfuchs

Karolinska Institutet https://orcid.org/0000-0001-6001-7240

Carl Sellgren ( $\square$ carl.sellgren@ki.se )

Karolinska Institutet

\section{Article}

Keywords: SARS-CoV-2, brain organoids, neuropsychiatric manifestations

Posted Date: August 6th, 2021

DOI: https://doi.org/10.21203/rs.3.rs-724318/v1

License: (c) (i) This work is licensed under a Creative Commons Attribution 4.0 International License. 



\section{SARS-CoV-2 Neurotropism and Single Cell Responses in Brain Organoids Containing Innately Developing Microglia}

Samudyata $^{1 \#}$, Ana Osório Oliveira ${ }^{1 \#}$, Susmita Malwade ${ }^{1}$, Nuno Rufino de Sousa ${ }^{2}$, Sravan K

Goparaju ${ }^{1}$, Funda Orhan ${ }^{1}$, Laura Steponaviciute ${ }^{2}$, Steven D Sheridan ${ }^{3}$, Roy H. Perlis ${ }^{3}$, Antonio Rothfuchs $^{2}$, Carl M. Sellgren ${ }^{1,4 *}$

${ }^{1}$ Department of Physiology and Pharmacology, Karolinska Institute, Stockholm, Sweden.

${ }^{2}$ Department of Microbiology, Tumor and Cell Biology, Karolinska Institute, Stockholm, Sweden.

${ }^{3}$ Center for Genomic Medicine and Department of Psychiatry, Massachusetts General Hospital, Boston, MA, USA.

${ }^{4}$ Centre for Psychiatry Research, Department of Clinical Neuroscience, Karolinska Institutet \& Stockholm Health Care Services, Stockholm County Council, Karolinska University Hospital, Stockholm, Sweden.

\# These authors contributed equally to this work

*To whom correspondence should be addressed: Carl M Sellgren, Dept. of Physiology and Pharmacology, Biomedicum C5, Solnavägen 9, 17165 Solna, Sweden. Phone: +46 (0) 70-212 72 87. Email: carl.sellgren@ki.se 


\begin{abstract}
Neuropsychiatric manifestations are common in both acute and post-acute phase of SARS-CoV-2 infection, but the mechanism of these effects is unknown. Here, we derive human brain organoids with innately developing microglia to investigate the cellular responses to SARS-CoV-2 infection on a single cell level. We find evidence of limited tropism to SARS-CoV-2 and observe extensive neuronal cell death that also include non-infected cells. Single cell transcriptome profiling reveals distinct responses in microglia and astrocytes that share features with cellular states observed in neurodegenerative diseases, includes upregulation of genes with relevance for synaptic stripping, and suggests altered blood brain barrier integrity. Across all cell types, we observe a global translational shut-down as well as altered carbohydrate metabolism and cellular respiration. Together, our findings provide insights into cellular responses of the resident brain immune cells to SARS-CoV-2 and pinpoint mechanisms that may be of relevance for the neuropathological changes observed in COVID-19 patients.
\end{abstract}




\section{INTRODUCTION}

A significant proportion of patients infected with severe acute respiratory syndrome coronavirus 2 (SARS-CoV-2), the causative agent of coronavirus disease 2019 (COVID-19), display acute neurological and psychiatric symptoms ${ }^{1}$, with autopsy studies detecting the presence of SARSCoV-2 RNA and protein in brain tissue of around half of the examined COVID-19 patients, despite low expression in neural cells of the major receptor (angiotensin-converting enzyme 2; ACE2,) for SARS-CoV-2 entry. ${ }^{2}$ Survivors commonly report persistent central nervous system (CNS)-related symptoms, such as memory loss, sleep disturbances ${ }^{3}$, and depressive symptoms, ${ }^{4}$ and a subset demonstrate structural brain abnormalities. ${ }^{5}$ Further, the risk of receiving a neurological or psychiatric diagnosis related to cognitive impairment or behavioral abnormalities, including dementia, psychosis, and major depressive disorder, is increased up to at least 6 months after infection. ${ }^{6,7,3}$ Long-lasting neurobehavioral and cognitive impairments are also common features of other RNA viruses with a neuro-invasive potential and the CNS-damaging processes can also continue after virus elimination. ${ }^{8}$ So far, the mechanisms causing neurocognitive sequelae after a viral encephalitis are poorly understood, but recent studies in rodents suggest that microglia, the resident immune cells of the brain parenchyma, play a pivotal role by inducing neuronal apoptosis and stripping neurons of synaptic terminals through complement-dependent signaling. ${ }^{9,10}$

Reprogrammed induced pluripotent stem cells (iPSCs) from easily-accessible human somatic cells can be used to derive 3D cellular aggregates in vitro referred to as organoids, which aim to provide mechanistic models that more precisely capture the broad repertoire of cell-cell interactions and complex spatial patterns that define the functions of complex tissues and organs such as the human brain. ${ }^{11-13}$ Brain organoids have also been successfully employed to study neurotropism and 
neurotoxic effects of viruses such as the Zika virus, ${ }^{14,15}$ and now more recently SARS-CoV-2. ${ }^{16-}$

${ }^{20}$ However, as microglia are of non-ectodermal origin, ${ }^{21,22}$ and as the predominate germ layer fate in embryoid bodies is ectodermal, brain organoids typically lack innately developing microglia. This has limited the mechanistic understanding of microglial cellular responses in viral CNS infection, such as in COVID-19, as well as the understanding of the cellular responses of astrocytes and neurons in the context of surveying microglia. To address this limitation, we applied a recently-developed protocol that generates brain organoids containing innately-developing microglia, ${ }^{23}$ adapted it to miniaturized bioreactors, and used it to characterize the temporal dynamics of single cell responses in a human experimental model upon SARS-CoV-2 infection.

\section{RESULTS}

SARS-CoV-2 neurotropism and cell death in brain organoids containing resident immune cells

With modifications (see Methods and Supplementary Fig. 1a), the protocol reported by Ormel et al. ${ }^{23}$ was used to generate brain organoids. At day 66 , these undirected organoids were found to contain PAX6+ cells (neural progenitor cells; NPCs), $\beta$-tubulin+ cells (neurons), SOX10+ cells (neural crest), GFAP+ cells (astrocytes), and IBA1+ cells (microglia); Supplementary Fig. 1b-e. We exposed the organoids to active SARS-CoV-2 virus for $2 \mathrm{~h}$ at an estimated multiplicity of infection (MOI) of 0.3 , after which they were washed and transferred into fresh media to monitor the course of infection. Supernatants were harvested at 6, 24, 48, 72 hours post-infection (hpi) for viral replication assessment. Viral RNA-dependent RNA polymerase $(R d r p)$ and nucleocapsid $(N)$ transcription were measured in supernatants via RT-qPCR and we observed a time dependent increase for both the transcripts (Fig. 1a and Supplementary Fig. 2a), indicating that the virus is capable of initiating RNA replication of its genomic material within the cells of the organoid, but 
at a slower pace as compared to Vero-E6 cells (Supplementary Fig. 2a). To validate that SARSCoV-2 could assemble and release complete viral particles in the organoids, we performed plaque formation assays using supernatants from infected organoids and observed release of infectious viral particles as early as 6hpi, although the number of plaque forming units (PFUs) then promptly decreased (Fig. 1b).

At two time points (24 and 72hpi), we then fixed infected and uninfected organoids (day 66) for immunohistochemical (IHC) analyses. At 24hpi, a strong signal for the viral nucleocapsid protein (NP) was observed mainly in the periphery of the infected organoids, whereas at 72hpi, we observed NP staining in cellular cytoplasm or processes of infected cells (Fig. 1c). On average, around 3\% of the cells in the superficial layers of the organoids were found to be NP-positive at 72hpi (Supplementary Fig. 2c). Caspase3 (Casp3) staining revealed extensive cell death in the infected organoids as compared to the uninfected organoids, occurring already at 24hpi (Fig. 1d). Further, Casp3+ cells were not just limited to superficial layers like NP+ cells (Supplementary Fig. 2b), and the number of Casp3+ cells greatly exceeded the number of NP+ cells at both timepoints (Supplementary Fig. 2c-d), then indicating viral-related cell death beyond toxicity occurring as a result of direct infection.

Next, we investigated infectivity and cell death in the context of cellular identity. IHC staining on infected organoids at 72hpi showed viral NP overlapping with PAX6+, NeuN+ (neuronal lineage), and GFAP+ cells (astrocytic), in addition to SOX10+ cells (Neural crest/schwann cells), and OLIG2+ cells (glial progenitors); Fig. 2a-d). Casp3+ staining was foremost observed in neurons (Supplementary Fig. 2f), but also included other cell types (Supplementary Fig. 2g). In addition, 
we detected IBA1+ cells (i.e., microglia) that stained positive for NP (Fig. 2e) although the scarcity of these cells (slightly below $1 \%$ on average) obstructed meaningful quantifications. Therefore, we enriched for microglia from two infected organoids using magnetic-activated cell sorting (MACS) and CD11b beads. qRT-PCR on cell lysates then confirmed the presence of immature microglia (AIF1 expression, no TMEM119 expression; Supplementary Fig. 2e) including $N$ gene copies (Fig. 2f). Further, we derived induced human microglia-like cells (iMGs) in 2D-culture, ${ }^{24}$ and exposed these cells to live SARS-CoV-2 virus (MOI=0.01). At $24 \mathrm{hpi}$, we detected dsRNA+ cells (Fig. 2g), indicating the presence of virus inside iMGs.

\section{Single cell characterization of brain organoids containing resident immune cells}

Droplet-based encapsulation of single cells using the 10X Genomics chromium system allowed us to profile and compare transcriptomes of individual cells isolated from SARS-CoV-2 infected brain organoids as compared to uninfected control organoids. Given the extent of cell death in the organoids exposed to an estimated MOI of 0.3 , we decreased the MOI to 0.1. Further, to achieve more mature microglia, we cultured the organoids up to 160 days. Given the low microglia counts in day 66 organoids we also enriched for CD11b-positive cells (MACS). In this way we were able to obtain good-quality transcriptomic data for fresh cells isolated from three experimental conditions: uninfected control organoids (7257 cells), $24 \mathrm{hpi}$ organoids (15254 cells), and $72 \mathrm{hpi}$ organoids (2825 cells), respectively (Supplementary Fig. 3a). Then, we integrated pre-processed data from each condition and performed unsupervised graph-based clustering to obtain cellular clusters with similar transcriptomic identities across conditions (Fig. 3a, Supplementary Fig. 3b). Supervised inspection of top differentially expressed genes (DEGs) per cluster and comparison to transcriptomes of cells from the developing human brain confirmed clusters comprised of radial glia (RG) (cyclingRG: EGFR, TOP2A, MKI67 (Supplementary Fig. 3c); RG1: EGFR, OLIG2, 
OLIG1; RG2: NES, and oRG: HOPX, FABP7), intermediate progenitors (IPCs: PAX6, EOMES), immature (GAD2, PAX6) and mature neurons ( $D C X, S L C 17 A 7)$ as well as more mature microglia (AIF1, LAPTM5, P2RY12), astrocytes, (GFAP, AQP4), choroid-plexus cells (TTR), perivascular cells (DCN, LUM), and endothelial precursors (CLDN5) (Fig. 3b-c, Supplementary table 1a). We also confirmed the transcriptomic identity of microglia derived from organoids by correlating to single-cell data of primary microglia (Fig. 3d-e).$^{25-27}$

\section{Confirming the expression of SARS-CoV-2 entry factors}

We then assessed the expression of previously identified entry factors for SARS-CoV-2 in the generated single cell data. Cells belonging to choroid plexus and a subset of neurons (expressing midbrain dopaminergic markers) showed relatively higher $A C E 2$ expression, although overall basal RNA expression and protein levels were relatively low (Fig. 4a-b and Supplementary Fig.

3d). Similarly, proteases involved in viral S protein priming ${ }^{28}$ (TMPRSS2 and TMPRSS4) showed low expression, whereas $F U R I N^{29}$ and infection potentiating factor ${ }^{24}(N R P 1$, Neuropilin 1) had a wider cell type distribution which included astrocytes, perivascular cells, endothelial cells and microglia (Fig. 4b). Microglia also expressed other predicted entry factors (CTSL and CTSB, Cathepsin L and B), purported to act as substitutes for TMPRSS2 ${ }^{25}$ (Fig. 4b). Next, we identified infected cells by aligning cellular viral transcripts to the whole SARS-CoV-2 genome in the infected conditions. As expected, given the decreased MOI, the percentage of infected cells were low (0.1-0.2\% of sequenced cells), although we were able to qualitatively identify infected neurons, radial glia, perivascular and choroid plexus cell types. For further downstream analyses, we did not make any distinction between infected and non-infected cells, instead focusing on overall cellular responses in the infected conditions. 


\section{Microglial responses to SARS-CoV-2 infection}

To define microglial responses to SARS-CoV-2 exposure, we first performed pseudo-bulk differential gene expression testing across the three experimental conditions, i.e., the uninfected control condition, 24hpi and 72hpi. An unbiased hierarchical clustering approach generated two unique groups of DEGs (labeled module A and B). Module A comprised of genes whose expression on average decreased at $24 \mathrm{hpi}$ and then at $72 \mathrm{hpi}$ displayed a more pronounced increase, while module B genes on average displayed reduced expression at $24 \mathrm{hpi}$ and a more dramatic decrease at 72hpi (Fig. 4c). While module B was largely comprised of genes encoding for cytosolic ribosomal proteins (Supplementary Fig. 4a), we detected several interferon-stimulated genes (ISGs) in module A such as ISG15, MX1, MX2, OAS2, OAS3, RSAD2 and BST2 (Fig. 4c,

Supplementary Fig. 4b). Nonetheless, we detected no clear upregulation of type I or type II interferon genes (IFNA, IFNB) in microglia, at either 24hpi or at 72hpi, although several pathogen sensors and upstream effectors of interferon signaling were upregulated including nuclear factor kappa B (NFKB1) (Fig. 4d). At 24hpi, we detected upregulation of pathways (such as oxidative phosphorylation, release of calcium into cytosol, monovalent inorganic ion transport, lysosomal disruption and hypoxia; Supplementary Table 2) that can serve as activating stimuli for primed inflammasome components (NLRP3, PYCARD, CASP1), and are required for the production and maturation of pro-inflammatory cytokines (Fig. 4d). ${ }^{30}$ Taken together, this result suggests that microglia, without the support of infiltrating myeloid cells, respond with an incomplete antiviral type-I and type-II interferon response to SARS-CoV-2 exposure. 
In the infected conditions, we also observed a significant upregulation of pathways related to neurodegenerative diseases, including Alzheimer's disease (AD) and Parkinson disease (PD) (Supplementary Fig. 4c). This led us to more closely examine the microglial transcriptional state after exposure to SARS-CoV-2 in relation to previously described disease-associated microglial activation states. First, we compared SARS-CoV-2-exposed microglia to such activation states observed in experimental murine models. Injury responsive microglia (IRM) have been described in mice close to experimentally induced focal demyelination ( 7 days post lesion) and are to a large extent defined by upregulation of ISGs, ${ }^{31,32}$ while the disease-associated microglia (DAM) described in mouse models of AD and ALS are thought to restrict neurodegeneration and disease development. ${ }^{33}$ SARS-CoV-2 exposed microglia then displayed a significant enrichment for genes associated with the IRM activation state $\left(\mathrm{p}=6.9 \times 10^{-24}\right.$, Fig. $\left.4 \mathrm{e}\right)$ and less so for the unique DAM signature genes $(\mathrm{p}=0.11)$, while still displaying upregulation of genes implicated in $\mathrm{AD}$ such as $A P O E, C T S Z$ and $G R N$ with a non-significant decrease of core microglial homeostasis genes (P2RY12, CX3CR1, P2RY13, CD33, TXNIP; Supplementary Fig. 4d). In fact, APOE and CTSZ were observed to be commonly dysregulated across all three signatures (IRM, DAM and SARS$\mathrm{CoV}-2$ ), then suggesting unique as well as shared activation mechanisms across these microglial states.

Further, we compared the SARS-CoV-2 microglial transcriptomic signature to previously identified human microglial populations identified in autopsy and surgical brain tissues obtained from subjects in the Memory and Aging Project (MAP), ${ }^{34}$ and found an enrichment with genes in so-called interferon responsive microglia (cluster 4 in this dataset) (Fig. 4f), defined by ISG15 and with increased expression of multiple sclerosis (MS) and AD susceptibility genes. To exclude 
effects of tissue processing, we also compared microglia from the organoids with a cluster defined by general cellular distress due to tissue processing (cluster 3) but observed no enrichment (Fig. 4f).

Actin-cytoskeletal remodeling pathways, essential for promoting migration and phagocytosis, ${ }^{35}$ were also upregulated in microglia exposed to SARS-CoV-2 (Fig. 4g, Supplementary Fig. 4c). Consistent with this upregulation in microglia, and the extensive neuronal cell death in infected organoids, even non-infected neurons at $72 \mathrm{hpi}$ downregulated the expression of 'don't-eat-me' signals such as CD46 and CD200 (Supplementary Fig. 4d) ${ }^{36}$ Consequently, we also observed increased microglial expression of genes associated with microglia-mediated phagocytosis, ${ }^{37-40}$ such as $C D 68, T R E M 2, I T G B 5, C D 47, M S R 1, C A L R$, as well as genes previously identified to be directly involved in pathological synaptic stripping of viral encephalitis such as $C 3, C 3 A R 1$ and FCGR $3 A^{8}$ (Supplementary Fig. 4e).

In summary, this suggest that microglia in response to SARS-CoV-2 exposure adopt transcriptional states that are unique but also overlap with signals observed in neurodegenerative diseases, and that these states are likely to include both protective - e.g., clearing of debris and defense as well as detrimental - e.g., inducing excessive neuronal apoptosis and synaptic stripping responses.

Astrocytic responses to SARS-CoV-2 infection

In response to SARS-CoV-2, astrocytes displayed DEGs enriched for mechanisms involved in cell cycle, carbon metabolism and disorders such as Huntington's disease (HD) and PD (Fig. 5a). 
Already at 24hpi, three subclusters of astrocytes could be identified: AS-0, AS-3 and AS-4 (Fig. 5b). AS-0 cells expressed relatively higher levels of anti-viral ISGs such as IFITI, ISG15, and SCRG1, while AS-3 and AS-4 cells had a proliferative profile (TOP2A, MKI67) and exhibited lower expression levels of GFAP (Fig. 5b-d). Subcluster AS-2, comprising of cells sampled at 72hpi, showed elevated levels of GFAP and STAT3, indicative of reactive astrogliosis, ${ }^{41}$ along with phagocytosis related genes such as $M E G F 8$ and $A B C A 1$ (Fig. 5c). We also observed upregulation of metal ion (zinc, copper, iron) homeostasis pathways involving metallotheionins (MT2A, MT1X, MT1E) in AS-2 astrocytes at 72hpi (Fig. 5d).

In neurodegenerative diseases, astrocytes have been shown to express markers of a putative reactive neurotoxic state commonly referred to as $\mathrm{A} 1 .^{42}$ Given astrocytic DEGs from infected organoids were enriched for such diseases, we integrated single-cell transcriptomic data from A1 astrocytes (stimulated with IL-1A, TNF-A and C1q) ${ }^{43}$ and SARS-CoV-2 exposed astrocytes via canonical correlation analysis (CCA). Although the majority of SARS-CoV-2 exposed astrocytes (AS-0, AS-1, AS-2) clustered separately from A1 astrocytes, the proliferative clusters (AS-3 \& AS-4), as well as a subset of AS-0 astrocytes showed clustering similarities with a portion of A1 astrocytes (Fig. 5d). Furthermore, higher expression levels of metallotheionins, as in AS-2 cluster (72hpi), have been observed in astrocytes from HD patients. ${ }^{44}$ In summary, similar to the observed effects in microglia, we observe responses in astrocytes to SARS-CoV-2 exposure that share features with cellular states observed in neurodegenerative diseases, but also a strong temporal heterogeneity that includes unique cellular states. 
Signaling impacting barrier integrity and responses in choroid plexus cell types

Potential entry routes to the CNS for SARS-CoV-2 includes the blood-brain barrier (BBB) or the blood-cerebrospinal-fluid-barrier (BCSFB), of which both involve cell types such as endothelial cells, pericytes, astrocytes and choroid plexus-epithelium. Upon encountering pathogens, these barrier cells act in concert to activate and regulate signal transduction pathways that aid invasion of peripheral immune cells and restoring of CNS homeostasis. ${ }^{45}$ Correspondingly, at 24 hpi we found increased ligand-receptor communication between astrocytes, neurons, microglia, perivascular, and endothelial cells (Fig. 6a), whereas at 72hpi, both microglia and neurons significantly reduced their communication with most other cell types, while choroid plexus cell types gained interactions (Fig. 6b). Sub-clustering of choroid plexus related cells (Fig. 6c) showed two control enriched clusters (CP-0 and CP-2), consisting of genes involved in maintaining solute homeostasis (AQP1), barrier or extracellular matrix (ECM) integrity (TIMP1, LGALS1, COL1A2, COL2A1, COL3A1) and amyloid clearance (TTR, MT3), amongst others (Fig. 6d). At 24hpi, we observed an enrichment of metallotheionins (MT1M, MT1E, MT1X, MT1F, MT2A: CP-6), similar to what we observed in astrocytes at $72 \mathrm{hpi}$, whereas genes involved in viral defense response (SAMHD1), regulation of reactive oxygen species (ROS) (SOD3) and ECM organization (FBLN1, SYNE1) were enriched at 72hpi (CP-5) (Fig. 6d). In addition to choroid plexus, differential expression analysis showed significant increase in expression of matrix metalloproteases and ECM-regulatory enzymes (MMP14, MMP9, LOX) at 72hpi in microglia, astrocytes and perivascular cells, whereas endothelial cells reduced expression levels of solute carrier SLC2A1 (Fig. 6e), all in agreement with signaling that promotes compromised CNS barriers. Astrocytes, pericytes and choroid plexus related cells were also observed to downregulate VEGF signaling, a potent inducer of BBB permeability, at 24hpi but upregulated it at $72 \mathrm{hpi}$, coinciding with increased 
signaling for leukocyte chemotaxis and activation in astrocytes, as well as antigen processing and presentation in choroid plexus (Fig. 6e). In aggregate, this result suggests that cell types implicated in barrier functions change their communication upon SARS-CoV-2 infection, indicative of compromised integrity, as well as altered secretome and signaling with peripheral immune cells.

\section{Metabolic dysregulation across cell types}

Protein accumulation in the endoplasmic reticulum (ER) following viral infection or excessive production of secretory proteins can induce ER stress and initiate counter measures in the form of reduced translation and export to ER along with unfolded protein responses (UPRs) ${ }^{46}$ In exposed organoids, we detected an upregulation of pathways related to UPRs (IRE1, PERK), ER stress, proteasomal degradation and autophagy, among cell types such as microglia, astrocytes, choroid plexus and perivascular cells (Supplementary Table 2). Further, a partial translational inhibition could be observed in microglia (module B, as discussed before), as well as most of the other cell types (Supplementary Table 2, Fig. 4g). We also observed a downregulation of the ROS metabolism across cell types, which can lead to dysregulation of the antioxidant cellular systems and result in oxidative stress. ${ }^{47}$ SARS-CoV-2 then relies on excessive glucose for its replication, ${ }^{48}$ and shunts glucose from glycolytic pathways to fuel oxidative phosphorylation and ATP production in a process known as the Warburg effect. ${ }^{49}$ In our dataset, pathways pertaining to cellular respiration (oxidative phosphorylation, electron transport chain coupled to ATP synthesis) were enriched at $24 \mathrm{hpi}$ in neurons, while those of glucose and pyruvate metabolism were downregulated (Supplementary Table 2). Furthermore, transcription of glucose transporter, GLUT1 (SLC2A1), lactate dehydrogenase ( $L D H A)$ along with monocarboxylate transporter, MCT4 (SLC16A3), was downregulated in astrocytes at 24 and 72hpi, indicating deficits in glucose 
export and redox cycling necessary for neuronal energization ${ }^{50}$ (Supplementary Table 2). This suggests that following exposure to SARS-CoV-2, CNS cell types undergo major stress that results in metabolic changes which are likely to impact cellular communication such as the important interplay between astrocytes and neurons.

\section{DISCUSSION}

Using brain organoids with innately developing microglia, we observe limited tropism for SARSCoV-2 in NPCs, mature neurons, glial progenitors, as well as in glia of astrocytic, microglial, and schwann/oligodendrocyte lineage. Despite a low percentage of infected cells at a modest virus load, we observe extensive cell death that also occurs in uninfected neurons. Single cell transcriptome profiling reveals distinct responses in microglia and astrocytes but also cellular states that overlap with disease-associated phenotypes observed in neurodegenerative diseases, including upregulation of genes implicated in microglia-mediated synaptic stripping in postencephalitis models, and suggests compromised blood-brain barrier integrity. Across all cell types, we further observe a global translational inhibition as well as altered carbohydrate metabolism and cellular respiration.

Previous studies using undirected brain organoids to study SARS-CoV-2 have yielded variable results in terms of infectivity. ${ }^{16-20}$ To some degree, this discordance may be a result of differences regarding virus load, between-subject variability, as well as the cellular composition of the organoids. In this study, we observe that most cell types were sparsely infected at a modest virus load. In agreement with previous reports, ${ }^{19,51}$ we over time observe an increase in viral gene copies in the supernatants of infected organoids. Moreover, when we perform plaque formation assays 
using the same supernatants, the number of plaques per unit volume rapidly decreased at later time points. This suggests that the cells either have an inefficient assembly and shedding of viral particles, or that the massive cell death observed as early as 24hpi decreases the availability of viable cells to sustain the production of fully assembled viral particles.

Existing protocols for generating brain organoids have several constraints that also limit our interpretations. Importantly, brain organoids most closely resemble the developing fetal brain rather than the mature adult brain. In this context, we cannot exclude that important difference exists in the cellular responses and tropism between immature and mature brain cells, and primarily our model should be considered to recapitulate the responses to SARS-CoV-2 of the developing fetal brain. Nonetheless, the observe cellular responses that to a large degree are consistent with molecular mechanisms that pertain to the observed clinical phenotypes in the aftermath of COVID19 infection in adults. Further, it is unclear to what extent the used virus loads recapitulate in vivo exposure. To avoid supra-physiological exposure, we therefore use modest virus titers with estimated MOIs in between 0.1 and 0.3 . Finally, our model does not include peripheral immune cells and thus cannot capture effects related to infiltrating immune cells. However, by excluding infiltrating monocyte-derived cells the model is optimized for assessing microglial responses then avoiding difficulties with distinguishing these resident cells from infiltrating cells that can obtain a largely overlapping signal in the CNS environment.

In summary, we here provide an experimental approach for modeling viral encephalitis that includes tightly orchestrated responses of microglia and astrocyte in the context of neuronal circuits. Challenging this model with live SARS-CoV-2 virus, we observe initial glial responses 
to virus exposure suggesting that the resident immune cells of the brain can contribute to CNS damage through pathological processes such as synaptic stripping and neuronal apoptosis. A key next step will be to determine the clinical importance of such molecular processes, and specifically whether they may contribute to the neurocognitive and neuropsychiatric symptoms observed in a subset of COVID-19 patients. If so, these microglia-containing organoid models may provide an opportunity to evaluate microglia-targeted therapeutics aimed at minimizing or preventing these COVID-19 sequelae. 


\section{MATERIALS AND METHODS}

\section{Ethics}

All individuals signed a written informed consent before participating in the study, as approved by the Institutional Review Board of Partners HealthCare (Boston, MA, USA) and the Regional Ethical Review Boards in Stockholm, Sweden. All relevant ethical regulations were followed when performing the study.

Vero E6 cells

Vero E6 (ATCC-CRL-1586) cells were maintained in Dulbecco's modified eagle medium (DMEM, Cytiva) supplemented with $5 \%$ heat-inactivated fetal bovine serum (FBS, Cytiva), 100 $\mathrm{U} / \mathrm{mL}$ penicillin and $100 \mu \mathrm{g} / \mathrm{mL}$ streptomycin (Cytiva).

iPSC reprogramming

Two healthy human iPSC lines (males) were obtained from the MGH Neurobank. Briefly, iPSC colonies were obtained using mRNA reprogramming in a feeder-free culture system as described previously. ${ }^{52}$ Stable iPSCs were expanded in NutriStem XF medium (Biological Industries) and on biolaminin $521 \mathrm{LN}$-coated (BioLamina) plates. iPSCs were then purified using MACS with anti-TRA-1-60 MicroBeads (Miltenyi Biotec) on LS columns. All fibroblasts and iPSCs were screened and found negative for Mycoplasma and stained positive for pluripotency markers like octamer-binding transcription factor 4 (POU domain, class 5, transcription factor 1) and TRA-160. 


\section{Brain organoid cultures}

Undirected brain organoids were prepared from single cell suspension of human iPSCs as previously described. ${ }^{23,53}$ Briefly, embryoid bodies (EBs) were generated by seeding 9000 single cells in each well of a low attachment 96-well U-bottom plate with Y-27632 ROCK inhibitor (10 $\mu \mathrm{M})$ for one day. EB media was replaced on day 5 with Neural induction media in the same 96well plate. On day 10-11, EBs were embedded in 30 $\mu 1$ Matrigel (Corning) using sheets of dimpled parafilm and incubated for $20 \mathrm{~min}$ at $37^{\circ} \mathrm{C}$ as previously detailed. Matrigel embedded single EBs were then either transferred to a $24-w e l l$ plate in $3 \mathrm{ml}$ of expansion media per well, on an orbital shaker $(90 \mathrm{rpm})$, or to a 12-well miniaturized bioreactor (largely overlapping with the design proposed by Qian et al.), ${ }^{11}$ until further use.

\section{Induced microglia-like cells}

iMGs were derived from monocytes (donated from one healthy male) using established methods previously described in detail. ${ }^{24,52}$ Briefly, whole blood was collected into vacutainer cell preparation tubes containing sodium citrate as an anticoagulant (Becton, Dickinson and Company) and processed as per the manufacturer's instructions. Peripheral blood mononuclear cells (PBMC) were isolated, washed twice with PBS by centrifugation and suspended in heat-inactivated fetal bovine serum (FBS; Sigma) containing 10\% DMSO (Sigma). The cell suspension was then divided into aliquots, transferred into cryovials and cryopreserved by using a liquid nitrogen cooled freezer. Generation of induced microglia-like cells (iMG) from PBMCs were carried out using methods previously described, ${ }^{24}$ with minor modifications. Briefly, cryopreserved PBMC samples were transferred from liquid nitrogen freezer to a $37{ }^{\circ} \mathrm{C}$ water bath. Once the cell 
suspension had been thawed, it was gently pipetted into $10 \mathrm{ml}$ of pre warmed complete RPMI medium (CM) consisting of basal RPMI-1640 supplemented with 10\% heat-inactivated FBS and 1\% penicillin-streptomycin (P/S; Thermo Fisher Scientific). Following centrifugation (300 $g$ for 5 min at room temperature), the supernatant was discarded, and the cell pellet was resuspended in the appropriate volume of CM.

Isolated PBMCs were cultured at a density of $5 \times 10^{5}$ cells $/ 1 \mathrm{ml} \mathrm{CM}$ on $24-$ well plates coated with Geltrex (Thermo Fisher Scientific). After $24 \mathrm{~h}$ of incubation, the media was replaced with RPMI1640 supplemented with 1x glutamax (Life Technologies), 1\% P/S and $0.1 \mu \mathrm{g} \mathrm{ml}^{-1}$ of interleukin (IL)-34 (R\&D Systems) and $0.01 \mu \mathrm{g} \mathrm{ml}^{-1}$ of granulocyte macrophage colony-stimulating factor (GM-CSF; R\&D Systems). After 7 days, fresh media was added. At day 11, cells were used for infection.

\section{Virus isolate}

SARS-CoV2 (GenBank: MT093571.1), originally from the Public Health Agency of Sweden, was propagated on $90 \%$ confluent Vero E6 cells for 3 days at $37^{\circ} \mathrm{C}$. Cell debris were removed by centrifugation at $300 \mathrm{RCF}$ for 5 minutes and the viral supernatant was aliquoted into cryovials and stored at $-80^{\circ} \mathrm{C}$. Viral titers were quantified by PFU assay.

\section{Viral infection}

All experimental studies involving infectious SARS-CoV2 were performed within the biosafety level 3 (BSL3) facility at Karolinska Institute. From single cell dissociation experiments, approximately $1 \times 10^{6}$ cells were found to be present in a D65 organoid. Based on this, $3 \times 10^{5}$ 
$\mathrm{PFU} / \mathrm{ml}$ of SARS-CoV2 was used to infect organoids for $2 \mathrm{~h}$ (MOI 0.3 ) in a spinning bioreactor. Following viral exposure, the organoids were washed twice with PBS and transferred into fresh medium $(2 \mathrm{ml})$ to follow the course of infection. To monitor viral replication, $100 \mu \mathrm{l}$ of medium was collected at specified time points, centrifuged at $300 \mathrm{~g}$ for $5 \mathrm{~min}$ and the supernatant was collected in trizol for qPCR analysis. For single cell RNA sequencing (scRNA-seq), the viral load was reduced to $1 \times 10^{5} \mathrm{PFU} / \mathrm{ml}(\mathrm{MOI} 0.1)$ in order to reduce the percentage of cell death observed with the previous MOI.

\section{Plaque forming unit assay}

PFU assays was performed on 24-well cell culture plates seeded with $2 \times 10^{5}$ Vero E6 cells per well. A serial dilution of the inoculum medium (consisting of either the viral stock or experimental samples in DMEM) was prepared and $200 \mu \mathrm{L}$ used to infect each well. The plates were then incubated for 1 hour at $37^{\circ} \mathrm{C}, 5 \% \mathrm{CO} 2$. After the incubation period, the wells were washed twice with PBS and $1 \mathrm{~mL}$ of overlay medium (2:3 mix of 3\% high density carboxymethyl cellulose and complete DMEM medium) was added to each well and the plates incubated for 3 days at $37{ }^{\circ} \mathrm{C}$, $5 \% \mathrm{CO} 2$. The plates were subsequently inactivated with $1 \mathrm{~mL}$ of $10 \%$ formaldehyde solution overnight at RT, washed twice with PBS, stained with $200 \mu \mathrm{L}$ of crystal violet solution for $30 \mathrm{~min}$ at room temperature and the plaques counted.

\section{Immunohistochemistry}

Organoids were fixed with $4 \%$ paraformaldehyde for $20 \mathrm{~min}$ at RT followed by $24 \mathrm{~h}$ in $30 \%$ sucrose in PBS. Organoids were then embedded in OCT (VWR) and frozen at $-80^{\circ} \mathrm{C}$. Cryosections $(16$ um) of brain organoids were incubated with blocking solution for $1 \mathrm{~h}$ (10\% of Normal Donkey 
serum in $0.3 \%$ Triton $\mathrm{X}$ ) in $1 \mathrm{xPBS}$. Blocked cryosections were incubated with the respective primary antibodies, diluted in blocking solution and incubated overnight at $4^{\circ} \mathrm{C}$ : anti-ACE2 (rabbit, Nordicbiosite ASJ1B6222, 1:100), anti- $\beta$-III-tubulin (mouse, Promega G712A, 1:500), Cleaved caspase-3 (rabbit, Cell Signaling, 1:300), anti-PAX6 (mouse, Developmental Studies Hybridoma Bank, 1:100), anti-SOX10 antibody (goat, R\&D AF2864, 1:50), anti-OLIG2 (goat, R\&D AF2418, 1:100), anti-GFAP (mouse, Sigma G3893, 1:100), anti-IBA1 (goat, Abcam AB5076, 1:100), anti-IBA1 (rabbit, Wako 019-19741, 1:100) and SARS-CoV-2 (2019-nCoV) Nucleoprotein / NP Antibody (rabbit, Nordicbiosite 158-40143, 1:200). Cryosections were washed three times with 1 xPBS and incubated with the secondary antibody for $1 \mathrm{~h}$ at RT. Secondary antibodies (all conjugated to Alexa Fluor 488, 555 and 647) were purchased from Life Technologies and used at a 1:500 dilution. Then, samples were washed three times with (1xPBS), incubated for 5 minutes with fluorescent nuclear DAPI stain (VWR; 1:5000), and mounted with DAKO (Life Technologies). Samples were imaged using a LSM800 confocal microscope.

\section{Image quantification}

Immunofluorescence images were analyzed using the CellProfiler software to measure and classify cells according to the expression of the selected markers. First, the software was trained to automatically segment the images into: (1) cells (DAPI positive objects); (2) NP or casp3 objects, followed by relating objects (1) and (2). For cell type specific quantifications, (2) was related to (3) objects segmented by cell type specific markers stained by antibodies indicated above. Finally, the related objects were expressed as a percentage of DAPI positive cells. 
Quantitative PCR (qPCR)

RNA extraction was performed using DirectZol RNA-Miniprep Kit (Zymo Research Inc.), following the manufacturer's protocol. Samples collected in TRI reagent were applied to Zymospin columns. DNase I treatment was performed for samples with genomic DNA. Bound RNA was washed, diluted in $30 \mathrm{ul}$ nuclease-free water and stored at $-80^{\circ} \mathrm{C}$. Quality and concentration of extracted RNA was determined using a NanoDrop (Thermofischer Scientific). Total RNA was reverse-transcribed to cDNA using the High-Capacity RNA-to-cDNA Synthesis kit (Thermofischer Scientific) following manufacturer's protocol. Reverse transcription reactions were carried out in 20ul reaction volume containing lug of input RNA with the following thermal cycler conditions: $37^{\circ} \mathrm{C}(60$ minutes $)+95^{\circ} \mathrm{C}(5$ minutes $)+4^{\circ} \mathrm{C}(\infty)$. cDNA was further diluted to $1: 3$ and used as templates for PCR reactions.

PCR reactions were performed using the StepOnePlus ${ }^{\mathrm{TM}}$ Real-time PCR system (Applied Biosystems, Thermofischer Scientific) with PowerTrack ${ }^{\mathrm{TM}}$ SYBR Green Master Mix. Expression of viral mRNA was assessed by evaluating threshold cycle $(\mathrm{Ct})$ values. Relative expression levels of $\mathrm{N}$ gene in cell lysates were normalized against a housekeeping gene, GAPDH (glyceraldehyde3-phosphate dehydrogenase), according to the Delta delta Ct method. Absolute quantification of viral gene expression levels in the supernatant were performed using the standard curve method. Primer sequences used in this study are:

GAPDH Forward - GGTGGTCTCCTCTGACTTCAACA

GAPDH Reverse - GTGGTCGTTGAGGGCAATG

N gene Forward - CATTGGCATGGAAGTCACAC 
N gene Reverse - TCTGCGGTAAGGCTTGAGTT

RdRp Forward - CGCATACAGTCTTRCAGGCT

RdRp Reverse - GTGTGATGTTGAWATGACATGGTC

Dissociations for single cell RNA sequencing

Briefly, three organoids per condition (control, 24hpi, 72hpi) were washed twice with DPBS without ions and cut into small pieces using a sterile scalpel. Single cell suspension was generated using neural dissociation kit (Miltenyi Biotec) according to manufacturer's protocol. Microglia was enriched using CD11b magnetic microbeads (Miltentyi Biotec) using MACS. Viability and cell numbers were assessed for both MACS-enriched and flow-through fractions before proceeding with the 10x protocol.

\section{Single-cell RNA-sequencing}

Single cell suspensions were loaded onto a Chromium controller chip v3.1 (10X Genomics), with a target output of 6000-7000 cells per channel. GEM generation, barcoding, cDNA amplification and library preparation was performed using the Single-cell 3' Gel Bead and Library v3.1 kit (10X Genomics) at the Eukaryotic Single Cell Genomics facility (SciLifeLab, Sweden). Amplified cDNA and final libraries were evaluated on a Bioanalyzer for quality control and sequenced on Illumina NovaSeq 6000 platform. 


\section{Data processing}

Sequenced data was processed through the CellRanger Software (5.0,10X Genomics) and transcripts were aligned to a combined reference of Human GRCh38-3.0.0 and SARS-Cov-2 genome (GenBank: MT093571.1). Filtered feature-barcode matrices from CellRanger were further subjected to quality control measures where low-quality cells with $<200$ uniquely expressed genes and high percentage of mitochondrial reads $(>20 \%)$ were filtered out. Doublets were identified using scDblFinder package and removed with caution. Genes expressed in fewer than 5 cells were filtered out. Filtered count matrices were merged and analyzed downstream using the Seurat package. Expression data per experimental condition was normalized using the regularized negative binomial regression method implemented in SCTransform, while regressing out the difference between the G2M and S phase scores, which served as a confounding factor in our dataset. Anchors across conditions were identified using FindIntegrationAnchors function and samples were integrated using canonical correlation analysis (CCA) implemented in the IntegrateData function of Seurat. Linear data compression using principal component analysis (PCA) was performed on 3000 highly variable genes. Top 30 PCs were used as the input to perform non-linear dimensionality reduction using UMAP.

\section{Clustering and cell-type identification}

We constructed a k-nearest neighbor (KNN) graph based on Euclidean distance in 30 PCs and performed unsupervised graph-based clustering using the Louvain algorithm (modularity resolution $=0.8$ ). Preliminary clustering of 39,808 cells identified 21 clusters yielding a clear separation of neuro-ectodermal clusters. Non-neuroectodermal clusters that did not express known marker genes related to cell types present in the brain, were removed. The remaining 26,148 cells 
were then subjected to second-level clustering in an integrated space (resolution=0.6), yielding 16

final clusters. The clustering was visualized using UMAP embedding in two dimensions. Top differentially expressed genes conserved across conditions for the final clusters were identified using a non-parametric Wilcoxon rank-sum test in FindConservedMarkers function. P-values were adjusted based on Bonferroni correction and genes with at least $25 \%$ cluster-specific expression, $>0.25$ average log-fold change, and FDR $<0.01$ were chosen to identify the clusters. All cells were assigned scores based on cell type-specific gene modules to further identify broad cell types such as neurons, astrocytes, microglia and oligodendrocytes. A priori set of markers curated based on previous studies of cerebral organoids and developing fetal brain was explored to manually annotate the clusters. Additionally, we leveraged existing published fetal brain and organoid scRNA-seq datasets and undertook a correlation-based method (Spearman) to compare our annotated clusters to transcriptional profiles of previously annotated cell types.

\section{Differential expression testing and functional interpretation}

We performed differential gene expression analyses on each cluster across conditions on lognormalized values using the MAST package in R. DEGs were considered significant if their Benjamini-Hochberg-adjusted P-value was $<0.05$ and a $\log 2$ fold change was $>0.25$. Significant DEGs were used to perform GO term overrepresentation analysis using the enrichR package. Gene set enrichment analysis (GSEA) was performed on ranked DEGs using fgsea package for pathway analysis (KEGG, GO:BPdatabase). Gene sets were limited by minSize=3 and nPerm=10000. Normalized enrichment scores were calculated and plotted for pathways with adjusted p-values $<$ 0.05. Statistical significance of association of DEGs with gene signatures from published datasets was performed using Fischer's exact test to obtain a p-value $(<0.05$, adjusted with $\mathrm{BH}$ method) 
and Odds ratio (OR). Number of genes expressed in the respective cell type was used as the genomic background.

\section{Intercellular interaction}

Cellular crosstalk was inferred via ligand-receptor pair expression using CellphoneDB package (version 2) in python. Statistical analysis was performed within the package with default parameters (1000 iterations) and no subsampling was done.

\section{Statistics}

The assumptions of each used test were checked. All reported p-values are two sided and type of statistical test is reported in the figure legends or in the main text.

\section{DATA AND CODE AVAILABILITY}

Processing of data and downstream analysis was performed in R (version 4.0.3). Figures used in this manuscript were generated in Python (version 3.6.12). Raw single-cell RNA sequencing data is deposited into GEO database (XXXX). Source code to reproduce the findings are available in our repository on Github (https://github.com/SellgrenLab/organoid-Covid19). All other data is available from the corresponding authors upon request. 


\section{REFERENCES}

1. Ramani, A., Pranty, A. I. \& Gopalakrishnan, J. Neurotropic Effects of SARS-CoV-2 Modeled by the Human Brain Organoids. Stem Cell Reports vol. 16 373-384 (2021).

2. Matschke, J. et al. Neuropathology of patients with COVID-19 in Germany: a postmortem case series. Lancet Neurol. 19, 919-929 (2020).

3. Taquet, M., Geddes, J. R., Husain, M., Luciano, S. \& Harrison, P. J. 6-month neurological and psychiatric outcomes in 236379 survivors of COVID-19: a retrospective cohort study using electronic health records. The Lancet Psychiatry 8, 416-427 (2021).

4. Perlis, R. H. et al. Comparison of post-COVID depression and major depressive disorder. medRxiv Prepr. Serv. Heal. Sci. (2021) doi:10.1101/2021.03.26.21254425.

5. Lu, Y. et al. Cerebral Micro-Structural Changes in COVID-19 Patients - An MRI-based 3-month Follow-up Study: A brief title: Cerebral Changes in COVID-19. EClinicalMedicine 25, 100484 (2020).

6. Garrigues, E. et al. Post-discharge persistent symptoms and health-related quality of life after hospitalization for COVID-19. Journal of Infection vol. 81 e4-e6 (2020).

7. Al-Aly, Z., Xie, Y.\& Bowe, B. High-dimensional characterization of post-acute sequalae of COVID-19. Nature 1-6 (2021) doi:10.1038/s41586-021-03553-9.

8. Chhatbar, C. \& Prinz, M. The roles of microglia in viral encephalitis: from sensome to therapeutic targeting. Cellular and Molecular Immunology vol. 18 250-258 (2021).

9. Vasek, M. J. et al. A complement-microglial axis drives synapse loss during virus-induced memory impairment. Nature 534, 538-543 (2016).

10. Garber, C. et al. T cells promote microglia-mediated synaptic elimination and cognitive dysfunction during recovery from neuropathogenic flaviviruses. Nat. Neurosci. 22, 12761288 (2019). 
11. Qian, X. et al. Generation of human brain region-specific organoids using a miniaturized spinning bioreactor. Nat. Protoc. 13, 565-580 (2018).

12. Lancaster, M. A. et al. Cerebral organoids model human brain development and microcephaly. Nature 501, 373-379 (2013).

13. Pasca, A. M. et al. Functional cortical neurons and astrocytes from human pluripotent stem cells in 3D culture. Nat. Methods 12, 671-678 (2015).

14. Cugola, F. R. et al. The Brazilian Zika virus strain causes birth defects in experimental models. Nature 534, 267-271 (2016).

15. Brain Region-specific Organoids using Mini-bioreactors for Modeling ZIKV Exposure HHS Public Access. 165, 1238-1254 (2016).

16. Ramani, A. et al. $<\mathrm{scp}>\mathrm{SARS}</ \mathrm{scp}>-\mathrm{CoV}-2$ targets neurons of $3 \mathrm{D}$ human brain organoids. EMBO J. 39, (2020).

17. Jacob, F. et al. Human Pluripotent Stem Cell-Derived Neural Cells and Brain Organoids Reveal SARS-CoV-2 Neurotropism Predominates in Choroid Plexus Epithelium. Cell Stem Cell (2020) doi:10.1016/j.stem.2020.09.016.

18. Pellegrini, L. et al. SARS-CoV-2 Infects the Brain Choroid Plexus and Disrupts the Blood-CSF Barrier in Human Brain Organoids. Cell Stem Cell 27, (2020).

19. Zhang, B. Z. et al. SARS-CoV-2 infects human neural progenitor cells and brain organoids. Cell Research 1-4 (2020) doi:10.1038/s41422-020-0390-x.

20. Song, E. et al. Neuroinvasion of SARS-CoV-2 in human and mouse brain. J. Exp. Med. 218, (2021).

21. Schulz, C. et al. A lineage of myeloid cells independent of myb and hematopoietic stem cells. Science (80-. ). 335, 86-90 (2012). 
22. Ginhoux, F. et al. Fate mapping analysis reveals that adult microglia derive from primitive macrophages. Science (80-. ). 330, 841-845 (2010).

23. Ormel, P. R. et al. Microglia innately develop within cerebral organoids. Nat. Commun. 9, $1-14$ (2018).

24. Sellgren, C. M. et al. Patient-specific models of microglia-mediated engulfment of synapses and neural progenitors. Mol. Psychiatry 22, 170-177 (2017).

25. Nowakowski, T. J. et al. Spatiotemporal gene expression trajectories reveal developmental hierarchies of the human cortex. Science (80-. ). 358, 1318-1323 (2017).

26. Velmeshev, D. et al. Single-cell genomics identifies cell type-specific molecular changes in autism. Science (80-. ). 364, 685-689 (2019).

27. Hodge, R. D. et al. Conserved cell types with divergent features in human versus mouse cortex. Nature 573, 61-68 (2019).

28. Hoffmann, M. et al. SARS-CoV-2 Cell Entry Depends on ACE2 and TMPRSS2 and Is Blocked by a Clinically Proven Protease Inhibitor. Cell 181, 271-280.e8 (2020).

29. Johnson, B. A. et al. Loss of furin cleavage site attenuates SARS-CoV-2 pathogenesis. Nature 591, 293-299 (2021).

30. Guo, H., Callaway, J. B. \& Ting, J. P. Y. Inflammasomes: Mechanism of action, role in disease, and therapeutics. Nature Medicine vol. 21 677-687 (2015).

31. Sala Frigerio, C. et al. The Major Risk Factors for Alzheimer's Disease: Age, Sex, and Genes Modulate the Microglia Response to A $\beta$ Plaques. Cell Rep. 27, 1293-1306.e6 (2019).

32. Hammond, T. R. et al. Single-Cell RNA Sequencing of Microglia throughout the Mouse Lifespan and in the Injured Brain Reveals Complex Cell-State Changes. Immunity 50, 
253-271.e6 (2019).

33. Keren-Shaul, H. et al. A Unique Microglia Type Associated with Restricting Development of Alzheimer's Disease. Cell 169, 1276-1290.e17 (2017).

34. Olah, M. et al. Single cell RNA sequencing of human microglia uncovers a subset associated with Alzheimer's disease. Nat. Commun. 11, 1-18 (2020).

35. Ohsawa, K., Imai, Y., Sasaki, Y. \& Kohsaka, S. Microglia/macrophage-specific protein Ibal binds to fimbrin and enhances its actin-bundling activity. J. Neurochem. 88, 844-856 (2004).

36. Gardai, S. J. et al. Cell-surface calreticulin initiates clearance of viable or apoptotic cells through trans-activation of LRP on the phagocyte. Cell 123, 321-334 (2005).

37. Schaer, D. J., Alayash, A. I. \& Buehler, P. W. Gating the radical hemoglobin to macrophages: The anti-inflammatory role of CD163, a scavenger receptor. Antioxidants and Redox Signaling vol. 9 991-999 (2007).

38. Gold, L. I. et al. Calreticulin: non-endoplasmic reticulum functions in physiology and disease. FASEB J. 24, 665-683 (2010).

39. Canton, J., Neculai, D. \& Grinstein, S. Scavenger receptors in homeostasis and immunity. Nature Reviews Immunology vol. 13 621-634 (2013).

40. Józefowski, S. \& Kobzik, L. Scavenger receptor A mediates H 2 O 2 production and suppression of IL-12 release in murine macrophages . J. Leukoc. Biol. 76, 1066-1074 (2004).

41. O’Callaghan, J. P., Kelly, K. A., VanGilder, R. L., Sofroniew, M. V. \& Miller, D. B. Early activation of STAT3 regulates reactive astrogliosis induced by diverse forms of neurotoxicity. PLoS One 9, 102003 (2014). 
42. Sofroniew, M. V. Astrocyte Reactivity: Subtypes, States, and Functions in CNS Innate Immunity. Trends in Immunology vol. 41 758-770 (2020).

43. Barbar, L. et al. CD49f Is a Novel Marker of Functional and Reactive Human iPSCDerived Astrocytes. Neuron 107, 436-453.e12 (2020).

44. Al-Dalahmah, O. et al. Single-nucleus RNA-seq identifies Huntington disease astrocyte states. Acta Neuropathol. Commun. 8, 1-21 (2020).

45. Chen, Z. \& Li, G. Immune response and blood-brain barrier dysfunction during viral neuroinvasion. Innate Immun. 27, 109-117 (2021).

46. Zhang, K. \& Kaufman, R. J. Signaling the unfolded protein response from the endoplasmic reticulum. Journal of Biological Chemistry vol. 279 25935-25938 (2004).

47. Poljsak, B., Šuput, D. \& Milisav, I. Achieving the balance between ROS and antioxidants: When to use the synthetic antioxidants. Oxidative Medicine and Cellular Longevity (2013) doi:10.1155/2013/956792.

48. Ana Campos Codo, A. et al. Elevated Glucose Levels Favor SARS-CoV-2 Infection and Monocyte Response through a HIF-1a/ Glycolysis-Dependent Axis 11 Elevated Glucose Levels Favor SARS-CoV-2 Infection and Monocyte Response through a HIF1a/Glycolysis-Dependent Axis. Cell Metab. 32, 437-446.e5 (2020).

49. Stoolman, J. S. \& Chandel, N. S. Glucose Metabolism Linked to Antiviral Responses. Cell vol. 178 10-11 (2019).

50. Weber, B. \& Barros, L. F. The astrocyte: Powerhouse and recycling center. Cold Spring Harb. Perspect. Biol. 7, 20396-20397 (2015).

51. Tiwari, S. K., Wang, S., Smith, D., Carlin, A. F. \& Rana, T. M. Revealing Tissue-Specific SARS-CoV-2 Infection and Host Responses using Human Stem Cell-Derived Lung and 
Cerebral Organoids. Stem Cell Reports 16, 437-445 (2021).

52. Sellgren, C. M. et al. Increased synapse elimination by microglia in schizophrenia patientderived models of synaptic pruning. Nat. Neurosci. 22, 374-385 (2019).

53. Lancaster, M. A. \& Knoblich, J. A. Generation of cerebral organoids from human pluripotent stem cells. Nat. Protoc. 9, 2329-2340 (2014). 


\section{ACKNOWLEDGEMENT}

We thank the study participants. We are also grateful to the participant core facilities at Karolinska Institutet; The Biosafety Level (BSL)-3 laboratory at Biomedicum, BIC, and SciLife Lab. We are thankful to Judit Ozsvar, an intern at the Sellgren laboratory at time of the experiments, for her help with cell culture work, as well as to Gretchen Majkowitz for valuable feedback on the manuscript. This work was supported by grants from Hjärnfonden postdoktorala stipendier (S.: PS2019-0063), the Swedish Research Council (C.M.S.: 2017-02559), Karolinska Institutet (C.M.S.: KID), regional agreement on medical training and clinical research between Stockholm County Council (A.L.F, C.M.S.), One Mind Foundation/Kaiser Permanente (C.M.S.), and Marianne and Marcus Wallenberg Foundation (C.M.S.).

\section{AUTHOR CONTRIBUTIONS}

C.M.S, S, A.O.O, S.K.G, and S.M conceived the project. R.H.P and S.D.S provided iPSCs. A.O.O derived the organoids with help from S. N.R.S and S performed the infections and related assays supervised by A.R, with help from L.S. A.O.O optimized and performed IHCs, imaging and quantifications with S. S.K.G optimized the qPCR assays and performed them with S.M. F.O derived microglia for 2D assays. S.M designed the scRNA-seq experiments with inputs from $\mathrm{S}$ and C.M.S. Dissociations for scRNA-seq was performed by S and N.R.S. S.M analyzed scRNAseq data. S and S.M interpreted the data. S, S.M and C.M.S wrote the manuscript with inputs from the other co-authors.

\section{COMPETING INTEREST}

The authors declare no competing interests. 


\section{MAIN FIGURES}
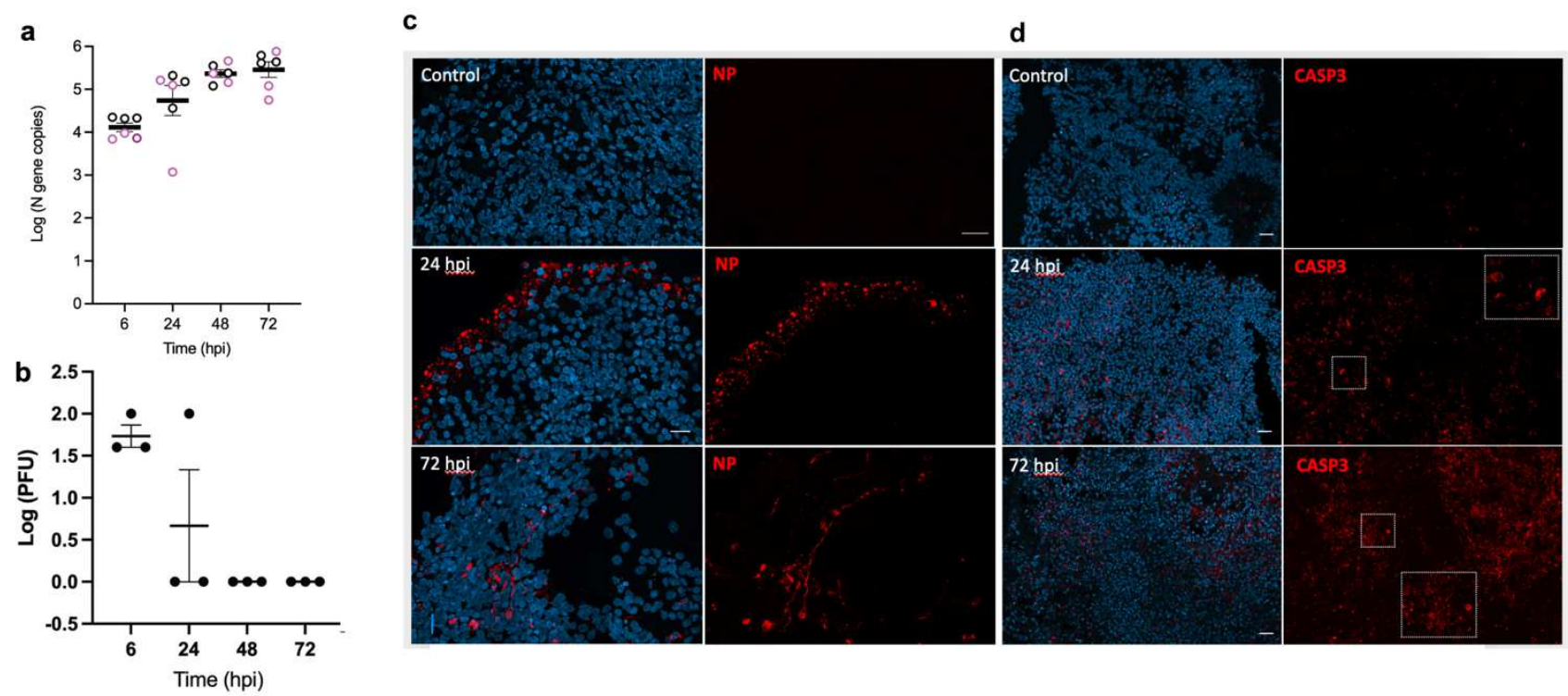

Figure 1: SARS-CoV-2 infection of brain organoids with innately developing microglia. Brain organoids infected with live SARS-CoV-2 virus was collected at different time points following exposure. (a) qPCR analyses on supernatants of infected brain organoids at day (D)66 ( $n=3$, black circle) and D120 ( $n=3$, magenta circle) showing log fold change in viral nucleocapsid $(N)$ gene copies, at different time points. Error bars indicate standard errors of the mean (SEM). (b) Log transformed PFUs of supernatants from infected brain organoids at different time points. Error bars indicate SEM. (c)-(d) Representative IHC tiled images showing staining of nucleocapsid protein (NP) (c), and Caspase 3 (CASP3) (d) in D66 infected brain organoids and uninfected control organoids. 

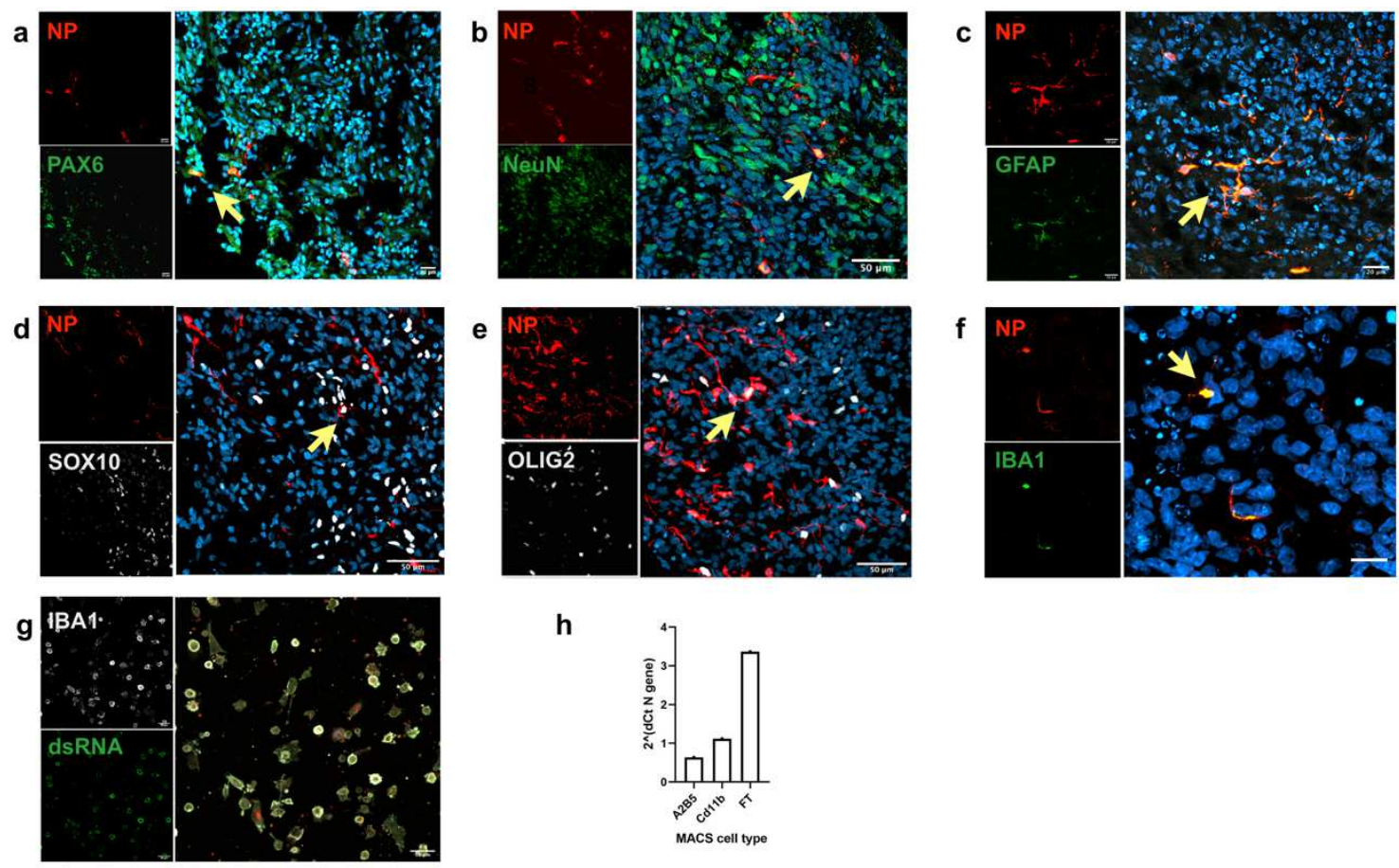

h

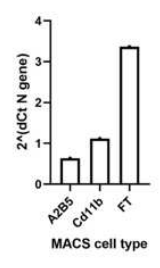

Figure 2: Cell type specific SARS-CoV-2 infection in brain organoids with innately developing microglia.40X confocal images showing colocalization of NP with (a) neuronal precursors (PAX6), (b) neurons (NEUN), (c) astrocytic cells (GFAP), (d) schwann cells (SOX10), (e) glial restricted radial glial cells (OLIG2) and (f) microglia (IBA1). (g) Induced microglia (iMG) from a healthy donor, infected with SARS-CoV-2 and stained for dsRNA (for the viral presence) and CASP3 (red), at 24hpi. (h) qPCR on cell lysate fractions obtained after magnetic cell sorting (MACS) with CD11b beads, (also shown is A2B5 radial glia enriched fraction) and flowthrough (FT), on infected organoids ( $\mathrm{n}=2$, pooled) showing presence of viral $\mathrm{N}$ gene copies. 

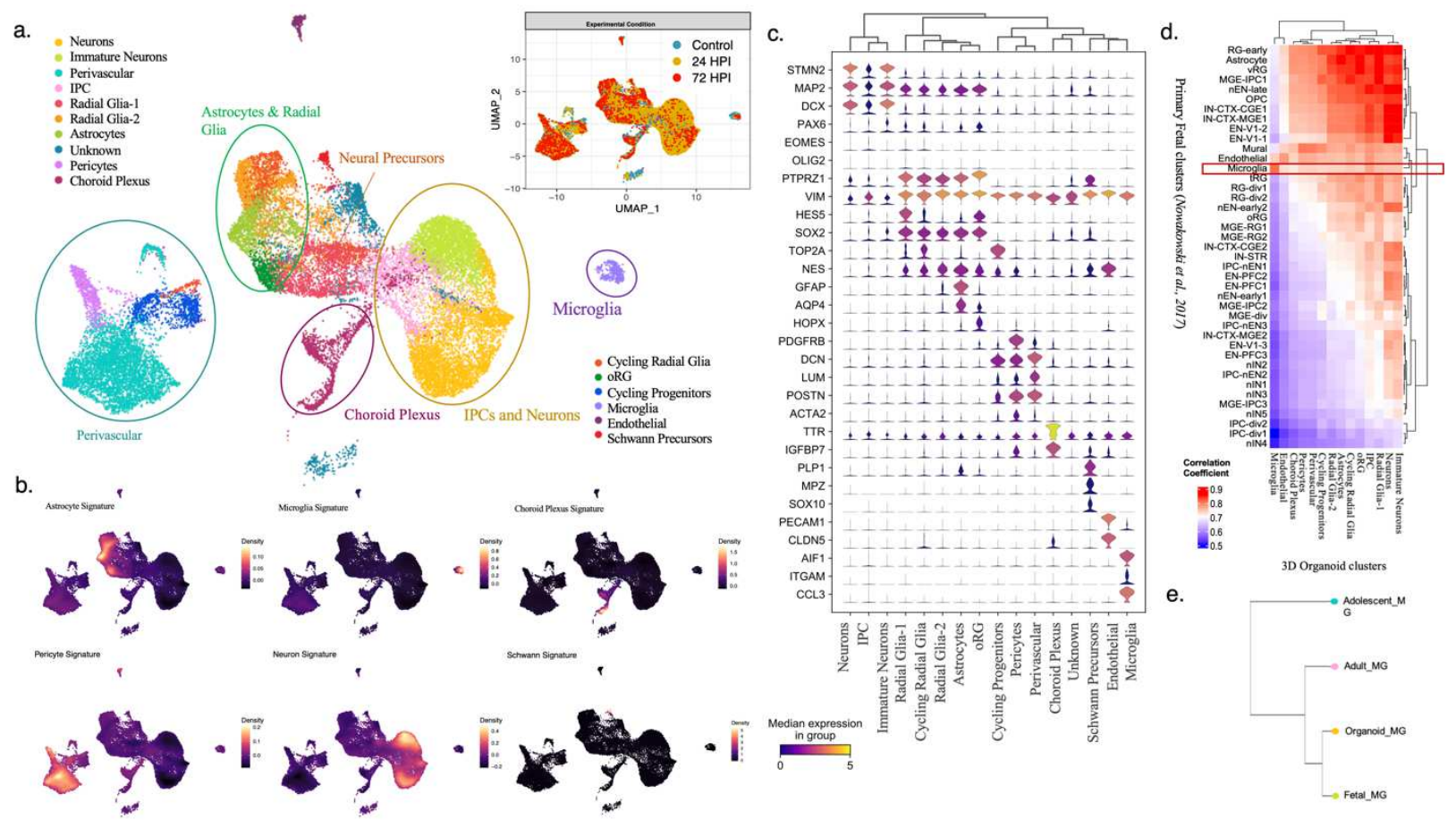

Figure 3. Single cell transcriptome profiling of brain organoids with innately developing microglia. Brain organoids $(\mathrm{n}=3)$ per experimental condition (control, 24 and $72 \mathrm{hpi}$ ) were dissociated into whole-cell suspensions and single cell RN sequencing libraries were generated using the droplet-based 10X platform. (a) UMAP plot of integrated dataset containing 25336 single cells from all three conditions depicting the presence of key neurodevelopmental cell types. Individual dots representing single cells are colored by the identified cell type. (b) Cell type classification obtained by scoring cells by their expression of known individual cell type signatures (Supplementary table 2) plotted as estimated joint density on a UMAP. (c) Stacked violin plots showing expression of cell type-specific markers (rows) across all clusters (columns). Top: dendrogram of cell type clusters (columns) obtained via unsupervised clustering. A list of all differentially expressed genes conserved across conditions 
for each cluster is provided in supplementary table1. (d) Spearman-ranked correlation of cerebral organoid clusters to reference transcriptome of primary fetal cortex (Nowakowski et al., 2017). (e) Dendrogram of microglial transcriptomic profiles comparing organoid-grown microglia to fetal (Nowakowski et al., 2017), adolescent (Velmeshev et al., 2019) and adult (Hodge et al., 2019) microglia. 


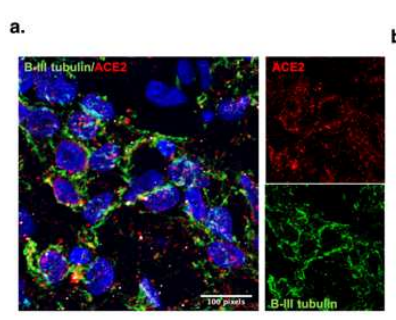

c.
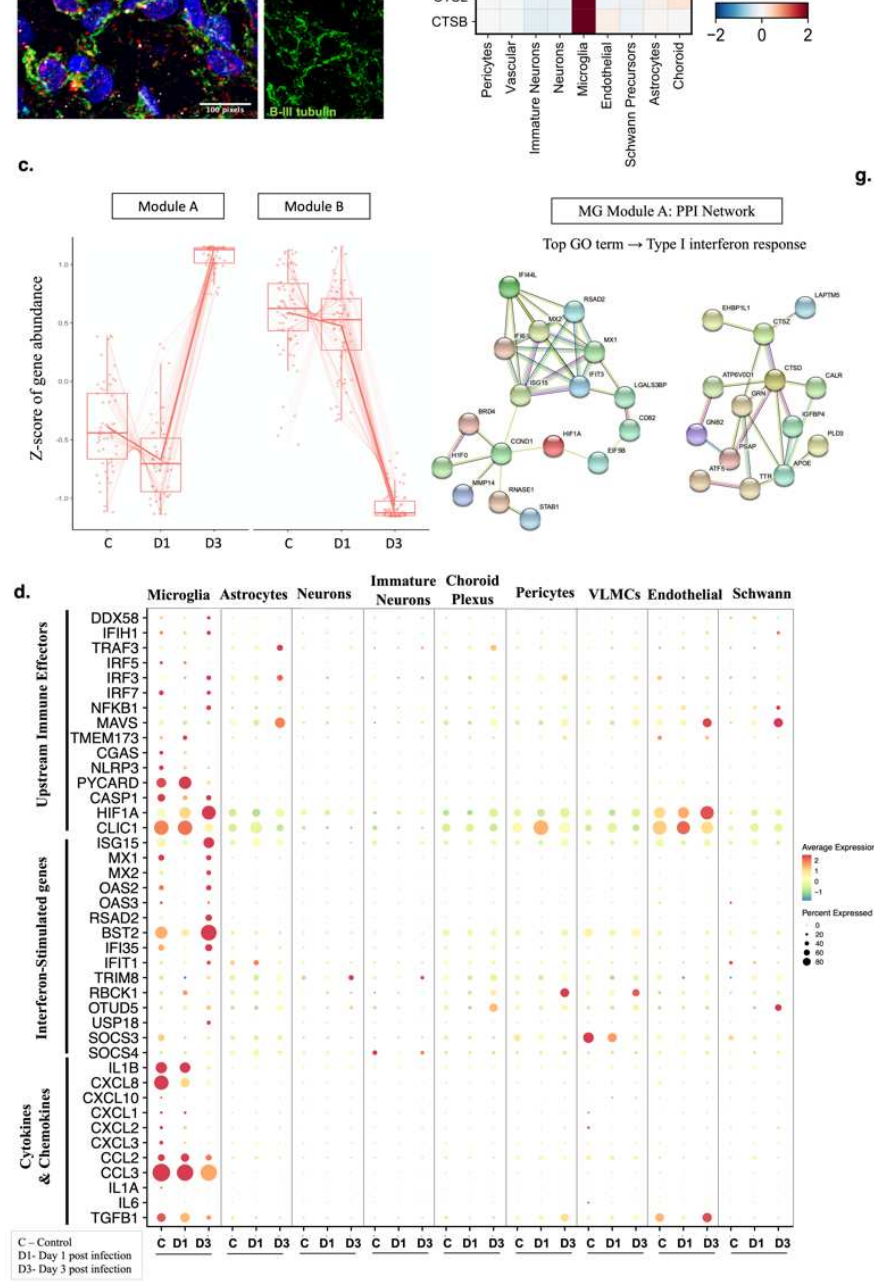

g.

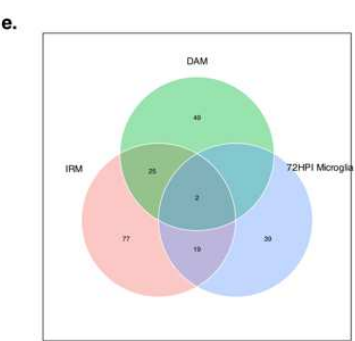

MG at 24 thpi N.S. N.S. N.S. N.S. N.S. N.S. N.S. N.S. N.S. MG at 72 hpi N.S. N.S. N.S. N.S. N.S. N.S. N.S. N.S

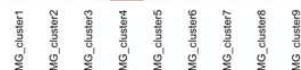

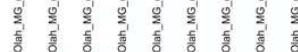

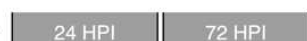

PROTEN LOCALIZATION_TO ENDOPLASMII_ RETICULUM
COTTANSLATIONAL PROTEIN_TARGETING_TO_MEMBRANE

VIRAA GENE EXPAESSION
PROTEN TARGETING TO MEMBANE

CEL JUNCTION ASEMBDY
EXTRACELLULAR MATRIX DISASSEMBIY

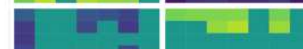

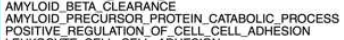

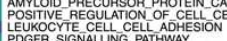

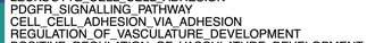

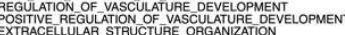

COLLAGEN_FiBRIL_OAGANIZATION

CELLULAR_RESPONSETOZ_ZINC
ZINC ION HOMEOSTASIS

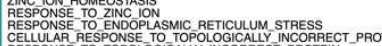

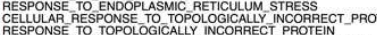
ENDOPLASMIC RETICULIM UNFOLLED PROTEIN_RESPONSE

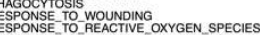

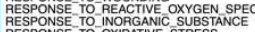
RESPONSE_TO_OXIDATIVE_STRESS
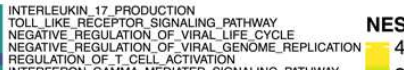

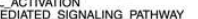
REGULATION OF CELL KILING SGETANG INTERLEUKKIN 1 BETTA PRODUCTION RESPONSE TO TYPE. INTERFEAO
DEFENSE RESPONS TO VIRUS _ - - - - - -4 MACOOPFAGE ACTIVATION

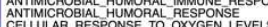

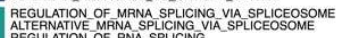
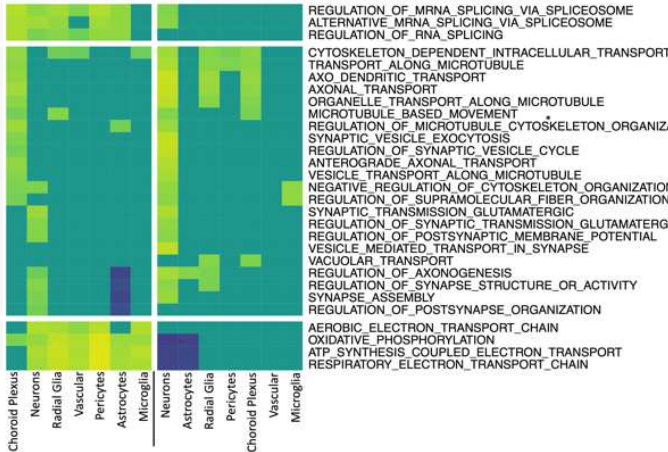

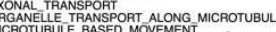
GULATION OF MECROOUVEUELEN CYTOSKKELETON ORGANIZATION AEGUATON OF SYNAPCTC VESICLE CYCLE
RETEROGRADE AXONAL TRANSPOBT ESSICLE TRANSPORT ALONG MICAOTUBU

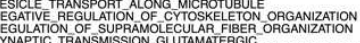
作

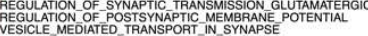

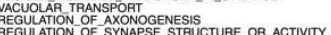
S AEROBIC ELECTRON_TRANSPORT_CHAIN 1) |III!||!!!!! (1)

Figure 4. Expression of SARS-CoV-2 entry factors in brain organoids and microglial responses to SARS-CoV-2. (a). IHC staining on D66 organoid showing ACE2 expression in BIII tubulin + neurons and OLIG2+ radial glia. (b) Heatmap showing relative mRNA expression levels of proposed cellular entry factors utilized by SARS-CoV-2 virus across cell types. (c) Modules of genes with similar expression behavior across conditions in microglia identified by pseudo-bulk differential gene expression analysis (using LRT implemented in 
DESeq2) - (left), [D1- 1 day/24 hpi; D3- 3days/72 hpi; C- control/uninfected]. (Right) Proteinprotein interaction (PPI) network of genes belonging to module A using STRING database with the top Gene Ontology term identified as 'Type I interferon response' (Adjusted pvalue $<0.05$ ). (d) Dot plot showing expression levels of neuro-immune related genes such as cytokines \& chemokines, interferon-stimulated genes and their upstream effector molecules across infected conditions for each cell type [D1- 1 day/24 hpi; D3- 3days/72 hpi; C- control/uninfected]. Color scale represents average log-scaled expression values across all cells, in each cluster. Size of the dot represents the percentage of cells in each cluster, expressing the gene. (e) Venn diagram of genes overlapping between gene signatures of Disease-associated microglia (DAM), injury-associated microglia (IRM) and SARS-CoV-2 infected microglia at 72hpi (* BH adjusted p-value < 0.005). (f) Heatmap of comparisons between SARS-CoV-2associated microglia at 24hpi \& $72 \mathrm{hpi}$ along with 9 human microglial clusters defined by Olah et al., 2020, colored by odds ratio (with p-values shown in red) from hypergeometric gene overlap testing (Fischer's exact test, Bonferroni correction, adjusted $p$-value $<0.05$ ). Transcriptomic gene signatures were defined as differentially upregulated genes with $\log 2 \mathrm{FC}>0.25$ and FDR $<5 \%$. (g) Heatmap showing gene set enrichment analyses (MSigDB GO:BP gene sets) of differentially expressed genes (DEGs) from 24hpi versus control (left) and 72hpi versus control (right) for each cell type. Pathways commonly dysregulated across most cell types were selected. All significantly altered pathways for individual cell types are listed in Supplementary table 2 (FDR $<5 \%$, Benjamini-Hochberg (BH) correction, NES- Normalized Enrichment Score). 


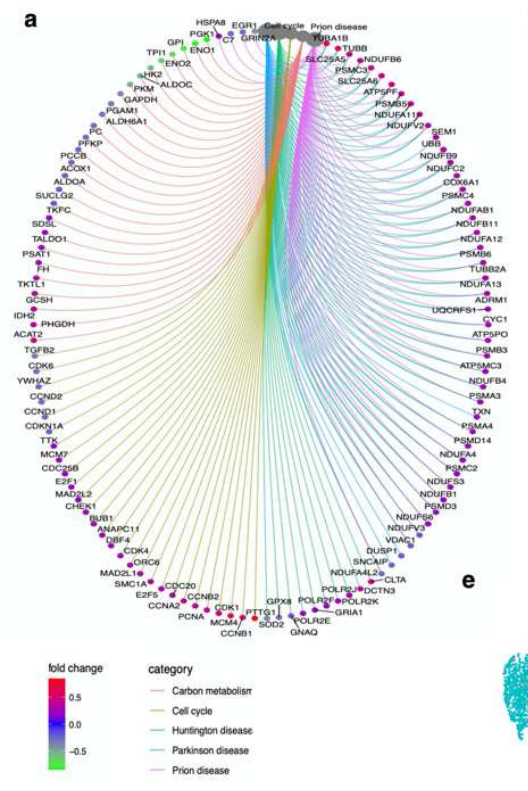

b Condition

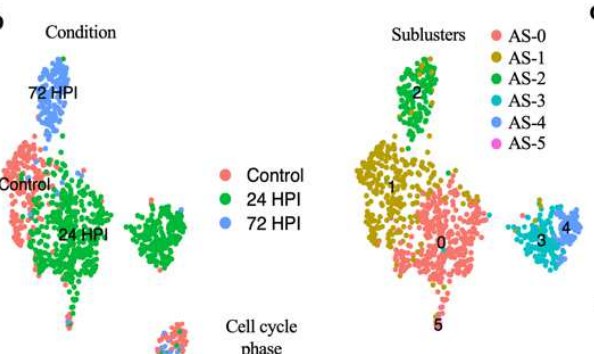

C Percent Expressed

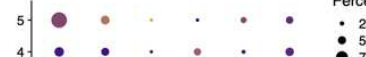

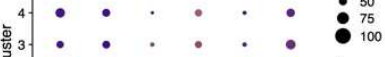

2. . A Average Expression

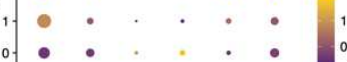

GFAP STÁT3 IFITI ISG'15 MEGFBABCA1

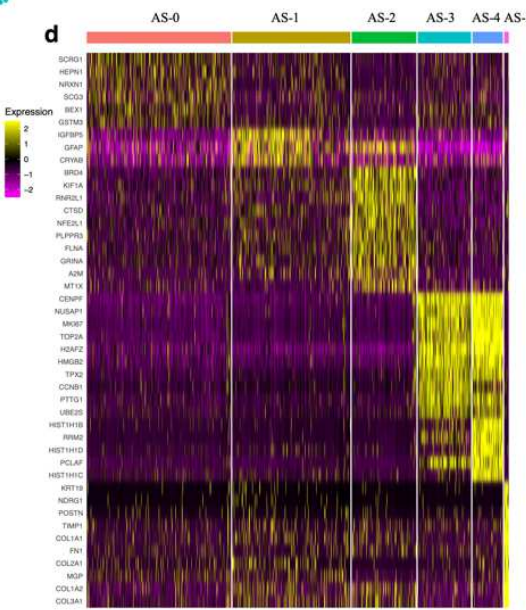

Figure 5. Temporal heterogeneity in astrocyte responses to SARS-CoV-2. (a) Differential expression analysis of SARS-CoV-2 exposed astrocytes versus control astrocytes, highlighted DEGs shown as a circular plot with top implicated pathways using overrepresentation test (FDR $<0.05$, Benjamini-Hochberg correction; Scale bar- Log2fold change values). (b) Cells belonging to the major astrocyte cluster, as shown in Fig 3A, were extracted and subjected to further unsupervised clustering. UMAP plot of astrocytes from all three conditions (left) shows 6 unique subclusters (AS-0, AS-2, AS-2, AS-3, AS-4 \& AS-5; middle) and the cellular cycle phase from each subcluster (right; clustering was performed upon regressing cell cycle effects). Each dot represents a single cell colored by the respective groups shown on the right side of each plot. (c) Dot plot of scaled average expression of genes involved in astrocytic engulfment across astrocyte subclusters (adjusted $\mathrm{P}<0.05$ ) (d) Astrocytic clusters are visualized as columns on the 
heatmap with relative expression levels of top differentially expressed marker genes (rows) of individual subclusters (using MAST test implemented in Seurat, adjusted p-value $<0.05$, Bonferroni correction). (e) UMAP plot of single-cell transcriptomic data of human iPSC-derived A1-astrocytes from Barbar et al., 2020, integrated with astrocytes from our study using canonicalcorrelation based-approach (CCA) (implemented in Seurat). Cells are colored by their respective dataset and experimental condition. 

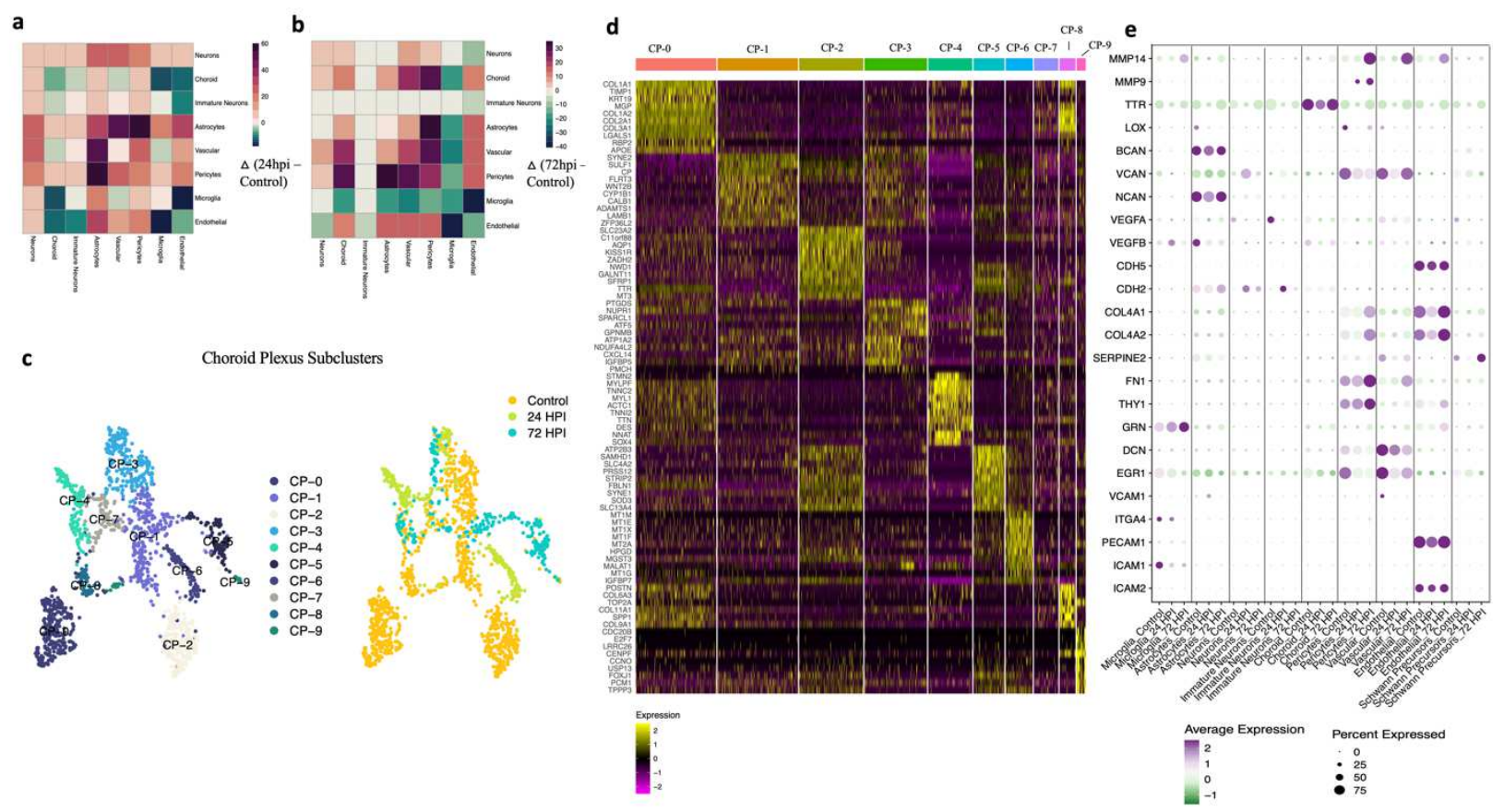

Figure 6. Choroid plexus cell subclusters in brain organoids exposed to SARS-CoV-2.

(a) Mapping intercellular interactions across cell types upon infection. Heatmap showing changes in the number of interactions between cell types at $24 \mathrm{hpi}$ and (b) $72 \mathrm{hpi}$ resulting from SARS-Cov2 infection. Ligand-receptor interactions were inferred in the single-cell transcriptomic data using CellphoneDB. Green depicts a negative difference indicating loss of interactions whereas purple depicts a positive change indicating gain of interactions between the cell type pair when compared to the control. (c) UMAP plot of all Choroid plexus cells colored by their identified subclusters (left) and experimental condition (right). (d) Heatmap showing scaled expression of top differentially expressed markers by choroid plexus subclusters. Top bar colored by each choroid plexus subcluster. High expression values shown in yellow while low expression values shown in purple. (e) Average-scaled expression of genes related to blood-brain-barrier integrity and function for different cell type clusters across control and infected conditions. 


\section{Supplementary Files}

This is a list of supplementary files associated with this preprint. Click to download.

- SupplfiguresNatComm.pdf

- SupplementaryTable1.xlsx

- Supplementarytable2.xlsx

- Supplementarytable3.xlsx 